
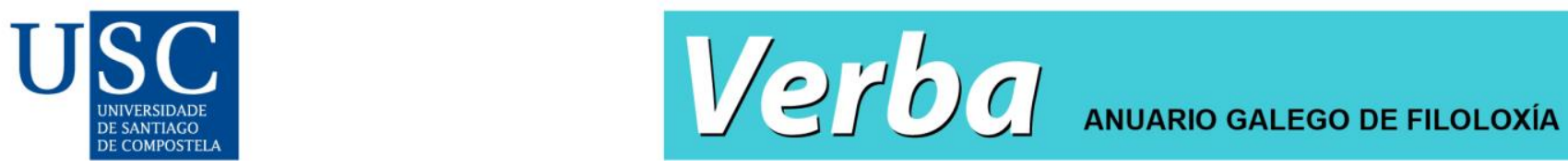

Verba: Anuario Galego de Filoloxía, 48, 2021. ISSN: 2174-4017

https://doi.org/10.15304/verba.48.6109

Artículos

\title{
Nombres escuetos en contextos no verídicos*
}

Bare nouns in non-veridical contexts

\author{
MATÍAS VERDECCHIA ${ }^{1}$ \\ 1IIF/SADAF/CONICET - Universidad de Buenos Aires, Argentina
}

Recibido: 22/06/2019; Aceptado: 18/05/2020

\section{Resumen}

En el presente trabajo analizamos la distribución de los nombres contables en singular sin determinante bajo el alcance de la negación en español (e.g., No leí libro mejor que este). Nuestra propuesta es que estos nombres están encabezados por un indefinido nulo $\delta$ que resulta referencialmente deficiente (Giannakidou 2011). En este sentido, estos SSNN se comportan como términos de polaridad que deben licenciarse en contextos no verídicos. Así, señalamos que su legitimación no se restringe únicamente al ámbito de la negación, sino que también puede darse en otras configuraciones no verídicas, tales como el antecedente de un condicional o una interrogativa total. Respecto a su significado, argumentamos que se trata de indefinidos antiespecíficos. Particularmente, sostenemos que no se interpretan como términos de elección libre, sino como indefinidos referencialmente vagos.

Palabras clave: nombres escuetos; no veridicidad; términos de polaridad; negación; antiespecificidad.

\section{Abstract}

In this paper I analyse the distribution of bare singular count nouns in the scope of negation in Spanish (e.g., No leí libro major que este). I propose that these nouns are headed by a null indefinite $\delta$, which is referentially deficient (Giannakidou 2011). Thus, these NPs behave as polarity items that must be licenced in non-veridical contexts. In consequence, I point out that they can be licensed not only in the scope of negation, but also in other non-veridical contexts, such as the antecedent of conditionals or polar questions. Regarding their meaning, I argue that they are anti-specific indefinites. Particularly, I claim that they do not behave as free choice items, but as referentially vague indefinites.

Keywords: bare nouns; non-veridicality; polarity items; negation; anti-specificity. 


\section{INTRODUCCIÓN}

Como fue largamente señalado por la bibliografía (Bosque 1996, Laca 1999, RAE y ASALE 2009), en español, la distribución de los nombres contables singulares sin determinante en posición de objeto se encuentra altamente restringida. A diferencia de lo que ocurre con los plurales desnudos (1) y los nombres de masa (2), la presencia de un nombre discontinuo en singular sin artículo da lugar a una oración agramatical.

(1) a. *Escribe artículo.

b. Escribe artículos.

(2) a. *Quiere libro.

b. Quiere agua.

Sin embargo, existen ciertos casos en los que podemos encontrar un nombre contable desnudo como complemento verbal. Uno de esos casos son aquellas configuraciones en los que el nombre se encuentra bajo el alcance de la negación ${ }^{1}$ (3) (Fernández Ramírez 1951, Lapesa 1996, Bosque 1980 y 1996, Sánchez López 1999, Costa 2007).

(3) a. No escribió artículo en el que no lo mencionara.

b. No leí libro mejor que Cien años de soledad.

c. No vi película de mi agrado.

En este trabajo, proponemos que esos SSNN están encabezados por un indefinido nulo $\delta$ que se caracteriza por ser referencialmente deficiente (Giannakidou 2011). De este modo, estos SSNN se comportan como términos de polaridad que se licencian en contextos no verídicos. Respecto a su significado, sostenemos que se trata de indefinidos antiespecíficos que dan lugar a vaguedad referencial.

El presente artículo se organiza de la siguiente forma. En la sección 2, describimos las características fundamentales de la construcción. En la sección 3, abordamos la cuestión del licenciamiento de estos nombres. En primer lugar, evaluamos la posibilidad de dar cuenta de su distribución desde un enfoque (pseudo)incorporacionista y concluimos que una explicación en estos términos resulta empíricamente inadecuada. En segundo lugar, consideramos la posibilidad de que estos nombres funcionen como palabras- $n$ y mostramos los límites de esta alternativa. En tercer lugar, introducimos nuestra propuesta. En concreto, sostenemos que estos nombres se comportan como términos de polaridad que deben legitimarse en contextos no verídicos. En la sección 4, abordamos la cuestión de su interpretación. De este modo, argumentamos que estas construcciones funcionan como indefinidos antiespecíficos referencialmente vagos. En la sección 5 , postulamos que estos SSNN se encuentran encabezados por un indefinido nulo $\delta$, que resulta referencialmente deficiente. Así, a partir de esta categoría vacía logramos vincular la necesidad de legitimación en contextos no verídicos y la lectura antiespecífica. Finalmente, en la sección 6, presentamos las conclusiones generales del trabajo. 


\section{BREVE DESCRIPCIÓN DEL FENÓMENO}

Los nombres desnudos que analizamos en este trabajo presentan ciertas características fundamentales que los distinguen de otros casos de nombres singulares contables sin determinante (veáse la nota al pie 1). Una de sus propiedades principales es la necesidad de que el SN se halle obligatoriamente bajo el alcance de la negación. En su ausencia, la oración resulta agramatical, como puede verse en los ejemplos de (4), que contrastan con los de (3).

(4) a. *Escribió artículo en el que no lo mencionaba.

b. *Leí libro mejor que Cien años de soledad.

c. *Vi película de mi agrado.

Vale aclarar que no es necesario que el operador negativo se encuentre superficialmente en la misma cláusula en la que se halla el SN. Como observamos en (5), con los predicados que admiten la negación anticipada ('Neg-raising'), como creer o imaginar, es posible que la negación se encuentre en una cláusula matriz y el SN, en una subordinada.

(5) a. No \{creo/*lamento\} que haya escrito artículo en el que no lo mencionara.

b. No \{imagino/*me gusta $\}$ que haya leído libro mejor que Cien años de soledad.

Además de la negación, otro elemento que resulta obligatorio es el modificador nominal. Dicho modificador puede ser de diversa naturaleza. Así, por ejemplo, puede tratarse de una cláusula relativa (6a), de una construcción comparativa (6b) o de una cláusula de participio (6c).

(6) a. No escribió artículo *(en el que no lo mencionara).

b. No leí libro *(mejor que Cien años de soledad).

c. No conozco persona *(nacida en Madrid).

Como señala Sánchez López (1999), la obligatoriedad del modificador diferencia estas construcciones de modismos negativos como los de (7). En esos casos, a pesar de que también deben encontrarse bajo el alcance de la negación, su estructura se encuentra, en mayor o en menor medida, fosilizada ${ }^{2}$ y su significado no se obtiene composicionalmente a partir de la mera combinación de sus constituyentes.

(7) a. No dijo palabra. (Cf. ${ }^{*}$ Dijo palabra.).

b. No probó bocado. (Cf. *Probó bocado.).

Si bien, entonces, el modificador nominal resulta obligatorio, cabe señalar que no cualquiera da lugar a una oración gramatical. En términos generales, debe tratarse de un modificador de carácter 
proposicional (e. g., una cláusula relativa). Así, por ejemplo, no es posible la presencia de un adjetivo relacional (8a) o calificativo (8b), o de ciertos SSPP (8c).

(8) a. *No leí novela policial.

b. *No leí novela atrapante.

c. *No leí novela de García Márquez.

En suma, la aparición de estos nombres escuetos parece estar sujeta a la presencia obligatoria de dos elementos: por un lado, la negación (o, como veremos más adelante, un entorno no verídico); por otro lado, el modificador proposicional. En la siguiente sección, discutiremos cuáles son las condiciones de legitimación para estos SSNN y, en consecuencia, ampliaremos el panorama empírico de esta construcción.

\section{SOBRE EL LICENCIAMIENTO}

En esta sección, abordamos la cuestión del licenciamiento de los nombres escuetos aquí estudiados. En primer lugar, introducimos los análisis (pseudo)incorporacionistas y mostramos que una explicación en estos términos resulta empíricamente inadecuada. En segundo lugar, evaluamos la posibilidad de que estos nombres se comporten como palabras- $n$ y señalamos los límites de esta explicación. Finalmente, presentamos nuestra propuesta. En concreto, argumentamos que estos nombres funcionan como términos de polaridad que se licencian en contextos no verídicos.

\subsection{Alternativas incorporacionistas}

Una de las líneas de investigación que se ha explorado de forma recurrente en la bibliografía es el análisis de los nombres escuetos en términos de incorporación nominal (Mithun 1984, Baker 1988). Dejando de lado los detalles técnicos particulares de cada teoría, podemos entender la incorporación nominal, en un sentido estricto, como un proceso morfosintáctico en el que un nombre se integra al verbo o preposición que lo selecciona, dando lugar a un predicado complejo. Típicamente, el nombre incorporado presenta un alto grado de defectividad, es decir, no contiene marcas de número, caso o definitud. A modo de ilustración, en (9) podemos observar un ejemplo del mohawk, una lengua que presenta incorporación nominal. Así, mientras que en (9a) encontramos el objeto en la posición de complemento verbal, en (9b) vemos su forma incorporada (Baker 1996, apud Murasugi 2014).

(9) a. Wa'-k-hnínu-' ne ka-nákt-a'

FACT-1sS-comprar-PUNC NE NsS-cama-NSF

'Compré la/una cama'

b. Wa'-ke-nakt-a-hnínu-' 


\section{FACT-1sS-cama- $\varnothing$-comprar-PUNC}

'Compré la/una cama'

Si bien este tipo de análisis se postuló originalmente para dar cuenta de las lenguas polisintéticas, diversos autores han intentado extender y adaptar estas propuestas al fenómeno de los nombres sin determinante en español, prestando especial atención a su combinación con verbos y preposiciones livianas (Wonder 1990, Masullo 1992 y 1996). Dado que en las construcciones que estudiamos en este trabajo también nos encontramos con un predicado cuyo objeto es un nombre escueto, cabe preguntarnos, entonces, si es plausible abordarlas bajo un enfoque incorporacionista. Creemos, sin embargo, que la respuesta a esta pregunta es negativa. Un análisis de estas estructuras en términos de incorporación se enfrenta con una serie de problemas fundamentales. En primer lugar, no es necesaria la adyacencia estricta entre el verbo y el nombre, es decir, puede haber sintagmas interviniendo entre ambos elementos (10). Esto resulta inesperado si asumimos que el verbo y el nombre se encuentran en una relación de integración léxica y forman un predicado complejo.

a. No encontré en toda la biblioteca libro que me gustara.

b. No escribió esta semana artículo en el que no lo mencionara.

En segundo lugar, como observamos en (11), en estas construcciones es posible topicalizar el objeto. Nuevamente, la posibilidad de mover el argumento interno a la periferia izquierda resulta problemático si suponemos que el nombre se encuentra integrado al predicado.
a. Libro mejor que este, no leí.
b. Persona más molesta que Juan, no conozco.

En tercer lugar, un análisis incorporacionista no podría dar cuenta de la obligatoriedad de la negación (4) ni de la del modificador (6): si el mecanismo de incorporación bastara para legitimar la presencia del nombre desnudo, no debería ser necesario ningún otro elemento para su licenciamiento. De este modo, a partir de lo anteriormente expuesto, consideramos que una explicación en términos de incorporación estricta resulta empíricamente inadecuada.

Ahora bien, en la literatura sobre nombres desnudos podemos encontrar una serie de propuestas que adoptan una versión "más débil" de la incorporación nominal, conocidas bajo el nombre de pseudoincorporación (Massam 2001, Dayal 2003 y 2011, Baker 2014). En términos generales, la pseudoincorporación involucra un conjunto de fenómenos que presentan las propiedades semánticas de la incorporación nominal, pero que difieren en los aspectos sintácticos. En este sentido, la pseudoincorporación se diferencia de la incorporación estricta en que el constituyente pseudoincorporado puede ser una frase, es decir, no debe ser necesariamente un núcleo. Así, por ejemplo, Massam (2001) señala que en niuano pueden "incorporarse" no solo nombres desnudos, sino también SSNN que incluyan adjetivos, frases preposicionales, cláusulas de relativo en subjuntivo, o incluso nombres coordinados. Además, a diferencia de la incorporación, la pseudoincorporación no requiere adyacencia estricta, de modo que es posible la inserción de elementos entre el predicado y el nombre. De esta forma, la pseudoincorporación no da lugar a 
complejos morfológicos, sino que los nombres pseudoincorporados pueden identificarse a partir de su posición sintáctica. En este sentido, los casos que presentamos en (10) y (11) en contra de un análisis incorporacionista no resultan problemáticos para un enfoque pseudoincorporacionista. Por otra parte, como sostienen Borik y Gehrke (2015), los nombres pseudoincorporados pueden presentar propiedades típicamente relacionadas con el nivel sintagmático, como por ejemplo, la marcación de número, como ocurre en hindi (Dayal 2003), o de caso acusativo, como se observa en húngaro y en griego (Gehrke y Lekakou 2013). No obstante, a pesar de que en algunas lenguas pueden tener marcas de número o de caso, los nominales pseudoincorporados siempre son morfosintácticamente deficientes en comparación con los argumentos no incorporados. Crucialmente, nunca presentan rasgos de definitud, de modo que no pueden ser considerados SSDD.

Desde el punto de vista semántico, los nombres pseudoincorporados se comportan de manera similar a los incorporados: tienen alcance estrecho obligatorio frente a operadores como la negación, presentan neutralidad de número y opacidad referencial, y, en muchos casos, describen una situación "institucionalizada" o estereotipada (Dayal 2003). De este modo, se suele afirmar que la pseudoincorporación es un proceso que se da en Forma Lógica y no en la sintaxis.

En el marco de estas propuestas, Espinal y McNally (2011) intentan dar cuenta de la distribución de los nombres singulares contables sin determinante en español y en catalán. La idea central de las autoras es que estos nombres desnudos son interpretados como argumentos sintácticos pero no semánticos. Respecto a su sintaxis, asumen que estos nombres aparecen en configuraciones como las de (12), es decir, en estructuras sintácticas mínimas, en el sentido de Hale y Keyser (1998), en las que el verbo solo toma como complemento al nombre escueto, dando lugar a un predicado complejo.

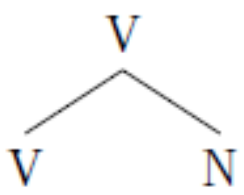

Además, siguiendo a Mateu (2002), proponen que estas estructuras están restringidas semánticamente, en tanto el verbo solo puede pertenecer a la clase de los have-predicates ${ }^{3}$ ('predicados del tipo de tener'). En este sentido, los nombres desnudos no pueden combinarse con predicados de tipo DO o CAUSE.

Respecto a su semántica, Espinal y McNally plantean que los nombres desnudos denotan propiedades de primer orden (i. e., son de tipo $\langle e, t\rangle$ ) y que funcionan como modificadores verbales. Para dar cuenta de su combinación con el verbo, las autoras proponen dos reglas: una regla léxica, que elimina el argumento interno del verbo, y una regla composicional, que permite combinar la propiedad denotada por el nombre escueto con el predicado.

A pesar de que esta teoría permite explicar satisfactoriamente casos como buscar casa o tener auto, creemos que no resulta adecuada para dar cuenta de la distribución de los nombres desnudos bajo el alcance de la negación. Por el contrario, la propuesta se enfrenta con numerosos problemas que nos llevan a creer que este fenómeno no constituye un caso de pseudoincorporación. En primer lugar, de acuerdo con Espinal y McNally, la pseudoincorporación en español solo puede 
estar licenciada por predicados del tipo de tener (have-predicates, como tener o poseer), predicados existenciales (ej. haber), ciertos verbos intensionales que implican una relación de 'tener en algún mundo posible relevante' (ej. necesitar, buscar), y ciertos predicados extensionales que suponen una relación posesiva o locativa (ej. ponerse, llevar, usar, comprar, encontrar, obtener, recibir). Como ya observamos en ejemplos previos, los casos aquí estudiados no se restringen al grupo de predicados señalados por las autoras: podemos encontrar nombres contables singulares sin determinante con verbos como escribir (3a), leer (3b) o ver (3c), que no se relacionan con las clases semánticas propuestas.

En segundo lugar, de acuerdo con Espinal y McNally, los nombres pseudoincorporados en español y en catalán son discursivamente opacos, es decir, no pueden funcionar como antecedentes de anáforas discursivas (e. g., pronombres personales como lo). En su propuesta, esto se sigue del hecho de que, en términos semánticos, estos nombres no constituyen verdaderos argumentos, sino que, como señalamos anteriormente, denotan propiedades ${ }^{4}$. En consecuencia, no pueden introducir referentes discursivos y, por lo tanto, no sirven como antecedentes. En (13), por ejemplo, observamos que en catalán el pronombre la no puede referir anafóricamente al nombre desnudo faldilla.

Avui porta faldilla. \#La hi vam regalar l'any passat.

hoy llevar.3SG falda la ella.DAT PAS.1PL regalar el.año pasado

'Hoy lleva falda. Se la regalamos el año pasado'

Si analizamos los casos de SSNN sin determinante en contextos de negación, en una primera aproximación notamos que en términos referenciales parecen comportarse como los nombres pseudoincorporados, dado que no pueden funcionar como antecedentes de pronombres anafóricos:

a. *No leí [libro mejor que Cien años de soledad] y loi recomendé.

b. *No escribió [artículo en el que no lo mencionara] y y loi publicó.

Sin embargo, rápidamente podemos comprobar que los casos de (14) no resultan adecuados para evaluar la opacidad referencial de estos SSNN. Como es sabido, los SSNN indefinidos bajo el alcance de la negación no pueden funcionar como antecedentes de anáforas discursivas (Karttunen 1976). Por ejemplo, en (15b), el pronombre nulo pro no puede referir al SN indefinido una hija.
a. Pedro tiene [una hija]i, proi se llama Juana.
b. *Pedro no tiene [una hija], proi se llama Juana.

En este sentido, en relación a los casos de (14), tampoco es posible la referencia anafórica incluso en los casos en los que, en lugar de un SN escueto, encontramos el indefinido negativo ningún:

*No leí ningún libroi mejor que este y loi recomendé. (Cf. [15a]). 
De este modo, la agramaticalidad de (14) parece deberse a motivos independientes a la presencia o ausencia del determinante en el SN (y, en consecuencia, a un posible proceso de pseudoincorporación nominal). Particularmente, como vimos en (15), es la negación la que imposibilita la referencia anafórica. Así, los datos de (14) no resultan metodológicamente adecuados para evaluar la posibilidad de que estos SSNN sin determinantes funcionen como antecedentes de pronombres personales. No obstante, podemos crear un escenario en el cual encontremos estos SSNN en ausencia de la negación. Como veremos más adelante, estos nombres escuetos pueden estar legitimados en otros contextos, como por ejemplo, bajo el alcance de un predicado seminegativo como dudar o en el antecedente de un condicional. Crucialmente, como observamos en (17), en ambas configuraciones los SSNN pueden actuar como antecedentes del pronombre $l o$, de modo que no resultan referencialmente opacos ${ }^{5}$.

(17) a. Dudo que haya leído [libro mejor que Cien años de soledad] y no te loi haya recomendado.

b. Si escribió [artículo en el que no lo mencionara]i, no loi leí.

Finalmente, una propuesta pseudo-incorporacionista como la de Espinal y McNally se enfrenta con el mismo problema que los enfoques incorporacionistas: no puede explicar por qué, en estos casos, son necesarios la negación (o en términos generales, como veremos más adelante, un contexto no verídico) y el modificador del nombre. Nuevamente, si la pseudoincorporación por sí sola legitimara estos nombres, es inesperado que ante la ausencia de la negación y del modificador estas oraciones resulten agramaticales.

En suma, a partir de las propiedades referenciales del SN y de las características léxicas del predicado, concluimos que estos casos de nombres escuetos bajo el alcance de la negación no pueden ser explicados bajo una propuesta pseudo-incorporacionista como la de Espinal y McNally. Sin embargo, hay una propiedad de los nombres pseudo-incorporados que sí parece estar presentes en estos casos: el hecho de tomar alcance estrecho frente a operadores como la negación (18).

(18) No leí libro mejor que Cien años de soledad.

a. Alcance estrecho: Neg > SN

(i. e., el hablante no leyó ningún libro mejor que Cien años de soledad)

b. *Alcance amplio: $\mathrm{SN}>\mathrm{Neg}$

(i. e., hay un libro mejor que Cien años de soledad que el hablante no leyó)

A pesar de que este fenómeno a priori parecería ser un argumento a favor de un análisis pseudoincorporacionista, puede recibir una explicación independiente, como mostraremos en el apartado 4. En este sentido, sostendremos que este comportamiento se deriva del hecho de que estos nombres sean antiespecíficos referencialmente vagos. 


\subsection{Nombres escuetos como palabras-n}

Si bien los sintagmas nominales sin determinante que estudiamos en este trabajo han llamado la atención de diversos gramáticos del español al menos desde Fernández Ramírez (1951), a nuestro saber, no se ha propuesto en la bibliografía ningún análisis sistemático de estas construcciones. No obstante, distintos autores han sugerido posibles explicaciones generales que asemejan el comportamiento de estos SSNN al de las palabras negativas. Así, por ejemplo, Bosque afirma:

[...] una posibilidad sería sostener que dichos SSNN están contenidos en una proyección cuantificativa encabezada por un cuantificador nulo inespecífico que cae bajo el ámbito de la negación. La relación entre este Q nulo y la negación sería similar a la que existe entre los términos de polaridad negativa (TPN) como nada, nadie, nunca, etc. y el inductor negativo que los legitima. (Bosque 1996: 39)

Retomando las palabras de Bosque, cabe preguntarnos hasta qué punto estos SSNN se comportan de manera similar a los términos negativos como nada o nadie. Exploremos una primera posibilidad: los SSNN considerados pertenecen a la misma clase natural que estas palabras negativas. Siguiendo la terminología de Laka (1990), llamaremos palabras-n a estos elementos (e. g., nada, nadie, nunca, etc.) y adoptaremos la siguiente definición de Giannakidou (2006):

Palabra- $n^{6}$ (Giannakidou 2006)

Una expresión $\alpha$ es una palabra-n si y solo si:

a. $\alpha$ puede ser usada en estructuras que contengan una negación oracional u otra expresión- $\alpha$, dando lugar a una lectura equivalente a una única negación lógica; y

b. $\alpha$ puede funcionar como un fragmento negativo en respuestas (i. e., sin la presencia de un elemento negativo explícito).

En una primera aproximación, podemos encontrar algunas similitudes entre las palabras-n y los SSNN desnudos: en ambos casos, es necesaria la presencia de la negación oracional (i. e., 'no') para legitimar la palabra-n o el SN en posición de objeto:
a. *(No) vi nada.
b. *(No) vi película mejor que esta.

Sin embargo, a pesar de esta aparente semejanza en el licenciamiento, la distribución de ambos elementos no es idéntica. En primer lugar, como afirma la definición (19), las palabras-n pueden funcionar como respuestas fragmentarias negativas sin la presencia explícita de una negación oracional (21a). Esto, sin embargo, no es posible con los nombres contables singulares sin determinante $(21 \mathrm{~b})$.
¿Qué viste?
a. Nada.
b. *Película mejor que esta. 
En segundo lugar, si bien tanto las palabras-n como los SSNN en cuestión deben estar legitimados por la negación, los SSNN pueden aparecer en otros contextos en los que las palabrasn no pueden hacerlo, como, por ejemplo, en interrogativas totales (22) o en oraciones que contengan ciertos modificadores cuantificativos seminegativos como rara vez (23):
a. ${ }^{*}$ ¿Viste nada?
b. ¿Viste película mejor que esta?
a. *Rara vez escribe nada7.
b. Rara vez escribe artículo en el que no lo mencione.

En tercer lugar, mientras que es posible topicalizar estos SSNN sin determinante, no es posible hacerlo con las palabras-n:
a. *Nada, no vi.
b. Película mejor que esta, no vi.

Finalmente, el mismo Bosque señala que el análisis de estos SSNN sin determinante como palabras-n no explica una diferencia fundamental en la legitimación de ambos elementos: las palabras-n, a diferencia de los SSNN escuetos, pueden estar legitimadas dentro de un SP por una negación externa (Benincà 1980).

(25) a. Nunca he llamado desde ningún teléfono que tuviera tantos botones.

b. *Nunca he llamado desde teléfono que tuviera tantos botones.

En conclusión, a pesar de que tanto las palabras-n como los SSNN desnudos pueden estar legitimados en contextos de negación, no constituyen el mismo fenómeno.

\subsection{Nombres escuetos como términos de polaridad}

Como mostramos en el apartado anterior, los SSNN desnudos que analizamos no tienen la misma distribución que las palabras-n. Sin embargo, a pesar de reconocer que no pertenecen a la misma clase natural, resta explicar por qué los SSNN también deben encontrarse bajo el alcance de la negación.
a. * Conozco ciudad más caótica que Buenos Aires.
b. No conozco ciudad más caótica que Buenos Aires.

Nuestra propuesta es que estos SSNN sin determinante son términos de polaridad (polarity items), en el sentido de Giannakidou (1999). Estos elementos se caracterizan por poder aparecer 
únicamente en contextos no verídicos. A los fines expositivos, adoptamos la definición de término de polaridad de (27) y la de no veridicidad de (28):

(27) Término de polaridad (Giannakidou 1999)

a. Un término de polaridad $\alpha$ es una expresión cuya distribución está limitada por su sensibilidad a cierta propiedad semántica $\beta$.

b. $\beta$ es al menos no verídico.

(28) Verdicidad, no veridicidad, antiveridicidad (Giannakidou 1998)

a. Una función $f$ es verídica si $f(\mathrm{p})$ implica $p$.

b. Una función $f$ es no verídica si $f(\mathrm{p})$ no implica $p$.

c. Una función $f$ es antiverídica si $f(\mathrm{p})$ implica no $p$.

De la definición de (28) se desprende que las funciones antiverídicas son un subconjunto de las no verídicas, ya que también satisfacen (28b). El ejemplo más claro de función antiverídica es la negación:
a. $p=$ Juan corrió.
b. $f_{\text {neg }}(p)=$ Juan no corrió.
c. No veridicidad:

$f_{\text {neg }}(p) \nRightarrow p$ (i. e., Juan no corrió no implica Juan corrió)

d. Antiveridicidad:

$f_{\text {neg }}(p) \Rightarrow \neg p$ (i. e., Juan no corrió implica Juan no corrió)

Esto permite explicar por qué las oraciones de (26) son agramaticales en ausencia de la negación: no se encuentran en un contexto no verídico y, por lo tanto, no pueden estar legitimados.

Ahora bien, la definición de no veridicidad, como señalamos previamente, es más amplia que la de antiveridicidad, ya que la primera implica a la segunda (pero no viceversa). En este sentido, esperaríamos que los SSNN sin determinante que estudiamos aparezcan en otros contextos además de la negación, a saber, en escenarios no verídicos que no necesariamente sean antiverídicos. Esta predicción se cumple: como podemos observar en el paradigma que presentamos a continuación, la distribución de estos SSNN sin determinante se extiende a otros contextos no verídicos, como el complemento de predicados seminegativos (31) y de la preposición sin (32), ciertos adverbios modales ${ }^{8}$ (33) o cuantificativos seminegativos (34), las interrogativas totales (35), y el antecedente o prótasis de las construcciones condicionales (36).

Negación

a. Bruno no escribió artículo en el que no lo mencionara.

b. No leí libro mejor que Cien años de soledad.

c. Juan no conoce ciudad más caótica que Buenos Aires. 
(31) Predicados seminegativos como dudar.

a. Dudo que Bruno haya escrito artículo en el que no lo mencionara.

b. Dudo que haya leído libro mejor que Cien años de soledad.

c. Dudo que Juan conozca ciudad más caótica que Buenos Aires.

(32) Preposición sin

a. Bruno se jubiló sin haber escrito artículo en el que no lo mencionara.

b. Me recibí sin haber leído libro mejor que Cien años de soledad.

c. Juan volvió de recorrer Latinoamérica sin haber conocido ciudad más caótica que Buenos Aires.

(33) Adverbios modales seminegativos como difícilmente

a. Bruno difícilmente haya escrito artículo en el que no lo mencionara.

b. Difícilmente haya leído libro mejor que Cien años de soledad.

c. Juan difícilmente haya conocido ciudad más caótica que Buenos Aires.

(34) Adverbios cuantificativos seminegativos como rara vez o pocas veces

a. Bruno rara vez escribió artículo en el que no lo mencionara.

b. Pocas veces leí libro mejor que Cien años de soledad.

c. Juan pocas veces visitó ciudad más caótica que Buenos Aires.

(35) Interrogativas totales

a. ¿Pedro escribió artículo en el que no lo mencionara?

b. ¿Leíste libro mejor que Cien años de soledad?

c. ¿Conocés ciudad más caótica que Buenos Aires?

Antecedente de condicional

a. Si Pedro escribe artículo en el que no lo mencione, va a tener problemas.

b. Si leí libro mejor que Cien años de soledad, no lo recuerdo.

c. Si conocés ciudad más caótica que Buenos Aires, decime cuál es.

El análisis de estos SSNN sin determinantes como términos de polaridad permite explicar, además, los contrastes previos de (22) y (23), que repetimos debajo. Como observamos previamente, estos SSNN, a diferencia de las palabras-n, pueden aparecer en contextos no negativos como las interrogativas totales o bajo el alcance de adverbios cuantificativos como rara vez. A la luz de las definiciones que adoptamos en (28), podemos explicar este contraste del siguiente modo: mientras que los SSNN sin determinante se licencian en contextos no verídicos, las palabras-n solo pueden estar legitimadas en contextos antiverídicos (e.g., la negación). En este sentido, la distribución de las palabras-n se encuentra más restringida que la de los SSNN sin determinante. 
a. ${ }^{*}$ ¿Viste nada?

b. ¿Viste película mejor que esta?

a. *Rara vez escribe nada.

b. Rara vez escribe artículo en el que no lo mencione.

Por otra parte, la necesidad de estos sintagmas de estar legitimados en entornos no verídicos permite diferenciarlos de otros casos de nombres escuetos que también son sensibles a la polaridad. Nos referimos en particular a las construcciones de alguno pospuesto (37a). En estos casos, encontramos un nombre desnudo acompañado por el indefinido alguno en posición posnominal. Crucialmente, este indefinido debe hallarse obligatoriamente bajo el alcance de la negación (37b).
a. No leyó libro alguno.
b. *Leyó libro alguno.

No obstante, a pesar de que en estos casos también hallamos nombres desnudos sensibles a la negación, consideramos que no pertenecen a la misma clase natural que los nombres escuetos aquí estudiados. Como señalamos previamente, estos últimos pueden legitimarse en contextos no verídicos. Sin embargo, en el caso de las construcciones con alguno pospuesto, su distribución se encuentra más restringida: solo puede estar licenciado en entornos antiverídicos ${ }^{9}$. De este modo, no pueden aparecer en configuraciones tales como las interrogativas totales (38) o ante la presencia de un adverbio modal como difícilmente (39).

(38) a. ¿Leíste libro mejor que este?

b. * ¿Leíste libro alguno?

(39) a. Difícilmente leí libro mejor que este.

b. *Difícilmente leí libro alguno.

\section{ANTIESPECIFICIDAD Y VAGUEDAD REFERENCIAL}

Hasta aquí hemos mostrado las condiciones de licenciamiento de estos SSNN. Particularmente, argumentamos que se trata de términos de polaridad que se legitiman en contextos no verídicos. Sin embargo, no hemos dicho nada sobre el significado de estas expresiones. Nuestra propuesta es que estos SSNN se interpretan como indefinidos antiespecíficos. Basándose en la noción de especificidad de Heusinger (2011), Giannakidou y Quer (2013) definen el concepto de antiespecificidad del siguiente modo: 
Ausencia de intención referencial por parte del hablante (i. e., el hablante no tiene en mente un valor específico del SN indefinido que utiliza).

Los indefinidos antiespecíficos, entonces, expresan la indeterminación referencial del SN, es decir, la ignorancia o la indiferencia del locutor acerca de la identidad exacta del referente. Dado que un indefinido antiespecífico supone que su valor referencial no se encuentra fijado, para que su uso sea feliz debe haber variación, esto es, el hablante debe considerar múltiples valores posibles. En español, como señalan Etxeberria y Giannakidou (2017), podemos reconocer por ejemplo el caso de algún. Para ilustrar su comportamiento, consideremos (41). En todos los casos, la segunda oración introduce un valor específico, ya sea por ostensión (41a), nombrándolo (41b), describiéndolo (41c), o pidiéndole al oyente que adivine cuál es el referente (41d). De este modo, el uso del indefinido antiespecífico de la primera oración se vuelve pragmáticamente infeliz, ya que el hablante sabe o cree saber su referente.
a. Tengo que quedar con algún profesor. \#Es aquel señor de allí.
b. Tengo que quedar con algún profesor. \#Se llama Bob Smith.
c. Tengo que quedar con algún profesor. \#Es el director del Departamento de Filosofía.
d. Tengo que quedar con algún profesor. \#¡Adiviná cuál!

Como podemos observar en (42), los SSNN que aquí estudiamos también presentan una interpretación antiespecífica:
a. Pocas veces conocí persona más molesta que Juan. \#Es aquel señor de allí.
b. Pocas veces conocí persona más molesta que Juan. \#Se llama Bob Smith.
c. Pocas veces conocí persona más molesta que Juan. \#Es el director del Departamento de Filosofía.
d. Pocas veces conocí persona más molesta que Juan. \#¡Adivina quién!

Cabe señalar que la antiespecificidad de estas construcciones parece deberse a la presencia del operador no verídico ${ }^{10}$. En este sentido, en estos mismos contextos, el indefinido una también presenta una interpretación no específica:
a. Pocas veces conocí una persona más molesta que Juan. \#Es aquel señor de allí.
b. Pocas veces conocí una persona más molesta que Juan. \#Se llama Bob Smith.
c. Pocas veces conocí una persona más molesta que Juan. \#Es el director del Departamento de Filosofía.
d. Pocas veces conocí una persona más molesta que Juan. \#¡Adivina quién!

Podemos encontrar evidencia adicional del carácter antiespecífico de estos SSNN en el hecho de que, cuando son modificados por cláusulas de relativo, estas deben hallarse en modo subjuntivo. Como es sabido, en español, el modo de las cláusulas de relativo está relacionado con la 
especificidad del SN. En términos generales, mientras que el modo indicativo permite designar entidades específicas que existen en un mundo concreto del universo del discurso, el modo subjuntivo indica que el SN es inespecífico (Pérez Saldanya 1999). Así, por ejemplo, en (44a) se hace referencia a un libro en particular, a diferencia de (44b), en la que el valor del libro no está fijado.

(44) a. Busco un libro en el que se analiza el modo en las oraciones de relativo.

b. Busco un libro en el que se analice el modo en las oraciones de relativo.

En este sentido, como afirmamos previamente, los SSNN aquí estudiados solo pueden estar modificados por cláusulas de relativo en subjuntivo:

a. *No escribió artículo en el que no lo mencionó.

b. No escribió artículo en el que no lo mencionara.

Otra prueba del carácter inespecífico de estas construcciones es la imposibilidad de aceptar cláusulas de relativo explicativas. Como señalan Brucart (1999) y Leonetti (1999), solo las relativas especificativas pueden presentarse con un antecedente inespecífico:
a. No he visto a nadie que piense así.
b. *No he visto a nadie, que \{piense/piensa $\}$ así.

De este modo, vemos que los SSNN escuetos en contextos no verídicos también rechazan la modificación por medio de cláusulas relativas explicativas:
a. *No escribió artículo mejor que este, en el que no lo \{mencionara/mencionaba\}.
b. *No visité ciudad mejor que esta, que \{fuera/es\} más caótica que Buenos Aires.

El hecho de que estos SSNN sean antiespecíficos nos permite dar cuenta de un fenómeno que mencionamos previamente y que tradicionalmente se vinculó con la pseudoincorporación: el alcance estrecho frente a operadores como la negación. Repetimos el contraste que señalamos anteriormente en (18):

(18) No leí libro mejor que Cien años de soledad.

\section{a. Alcance estrecho: Neg > SN}

(i. e., el hablante no leyó ningún libro mejor que Cien años de soledad)

b. *Alcance amplio: $\mathrm{SN}>\mathrm{Neg}$

(i. e., hay un libro mejor que Cien años de soledad que el hablante no leyó)

Este fenómeno se deriva directamente del hecho de que estos SSNN escuetos son siempre inespecíficos: nunca podrán tomar alcance por sobre la negación, ya que, si esto ocurriera, 
obtendrían una lectura específica. De este modo, podemos explicar este comportamiento sin la necesidad de asumir una propuesta pseudoincorporacionista.

Ahora bien, dentro de la clase de los indefinidos antiespecíficos, Giannakidou y Quer (2013) reconocen dos grupos: aquellos que expresan vaguedad referencial (referential vagueness) y los denominados términos de elección libre (free choice item). Si bien ambos tipos de indefinidos manifiestan la indeterminación referencial del SN, los segundos, a diferencia de los primeros, suponen la exhaustificación de todos los valores del dominio. En otras palabras, los términos de elección libre requieren que todos los posibles valores del dominio sean considerados, dando lugar a un efecto similar al de la cuantificación universal. En el caso de los indefinidos referencialmente vagos, por el contrario, solo encontramos una mera condición existencial, sin el requerimiento de la exhaustificación sobre el dominio.

En español, por ejemplo, podemos encontrar el indefinido referencialmente vago algún y el término de elección libre cualquier. Podemos ver el contraste entre ambos en escenarios en los cuales no todos los elementos del dominio de cuantificación son posibles referentes para el indefinido. Consideremos el siguiente caso de Alonso Ovalle y Menéndez Benito (2010):

(48) ESCENARIO. Estamos jugando a las escondidas y Juan está escondido. Pedro está convencido de que Juan no está en el baño ni en la cocina, pero por lo que sabe, Juan puede estar en cualquier otra habitación de la casa, o incluso afuera (por ejemplo, en la cochera).

(49) a. Juan puede estar en cualquier parte de la casa.

b. Juan puede estar en alguna parte de la casa.

Como podemos observar, dado el contexto de (48), (49a) resulta falsa y (49b), verdadera. La falsedad de (49a) se debe a que el término de elección libre cualquier lleva a considerar como posibles referentes a todos los elementos del dominio, en este caso, las partes de la casa. Sin embargo, de acuerdo al conocimiento de Pedro, ya sabemos que Juan no está en el baño ni en la cocina.

Consideremos ahora los SSNN escuetos en contextos no verídicos. Nuestra propuesta es que este tipo de construcciones son antiespecíficas referencialmente vagas. Para mostrar esto, retomamos dos pruebas utilizadas por Giannakidou (2018) para el griego, el coreano y el mandarín.

En primer lugar, mientras que los términos de elección libre pueden generar lecturas no selectivas ('just any') en las construcciones condicionales, los elementos referencialmente vagos no aceptan esta interpretación. En este sentido, observamos que los SSNN escuetos se comportan de manera análoga a los SSNN encabezados por algún:

(50) a. Si leés cualquier libro posterior a Pájaros en la boca, no estás siendo muy selectivo. b. \# Si leés algún libro posterior a Pájaros en la boca, no estás siendo muy selectivo. c. \# Si leés libro posterior a Pájaros en la boca, no estás siendo muy selectivo. 
En segundo lugar, como señalan Giannakidou y Quer (2013), los términos de elección libre, a diferencia de los indefinidos referencialmente vagos, no pueden encontrarse bajo el alcance de verbos modales universales. Crucialmente, en estos contextos sí es posible la presencia de un SSNN escueto.
a. \#Juan tiene que escribir cualquier artículo mejor que este.
b. Juan tiene que escribir algún artículo mejor que este.
c. ?Juan tiene que escribir artículo mejor que este.

En suma, en esta sección exploramos los aspectos interpretativos de los SSNN sin determinante en contextos no verídicos. Propusimos que se trata de indefinidos antiespecíficos y mostramos que no se comportan como términos de elección libre, sino como elementos referencialmente vagos. Un corolario de nuestra propuesta es que permite derivar los fenómenos de alcance estrecho bajo la negación sin la necesidad de postular un proceso de pseudoincorporación.

\section{INDEFINIDO NULO $\delta$}

En las secciones anteriores arribamos a dos conclusiones: por un lado, mostramos que las construcciones de nombre desnudo que aquí tratamos se licencian en contextos no verídicos; por otro lado, argumentamos que se interpretan como indefinidos antiespecíficos referencialmente vagos. Cabe preguntarse, entonces, qué relación existe entre estas dos propiedades.

Para responder esta cuestión, postulamos que estos nombres desnudos están encabezados por un indefinido nulo, al que llamaremos $\delta$. En términos sintácticos, estas construcciones presentan la siguiente estructura:

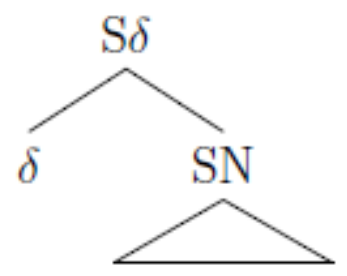

Siguiendo a Giannakidou $(2011,2001)$, proponemos que $\delta$ es un indefinido refencialmente deficiente. Esto significa que este elemento no puede introducir referentes discursivos en el contexto principal ${ }^{11}$, esto es, no asevera la existencia en el mundo actual wo del SN que selecciona. En este sentido, la variable que contiene no puede ser clausurada existencialmente a menos que se encuentre bajo el alcance de un operador no verídico, dado que, en estos entornos, no introducirá un referente discursivo en el contexto matriz (i.e., se bloquea la posibilidad de aseverar la existencia del SN en $\mathrm{w}_{0}$ ). Debido a la necesidad de este tipo de variables de hallarse en contextos no verídicos, Giannakidou las denomina variables dependientes ( $\mathrm{xd}$ ). 


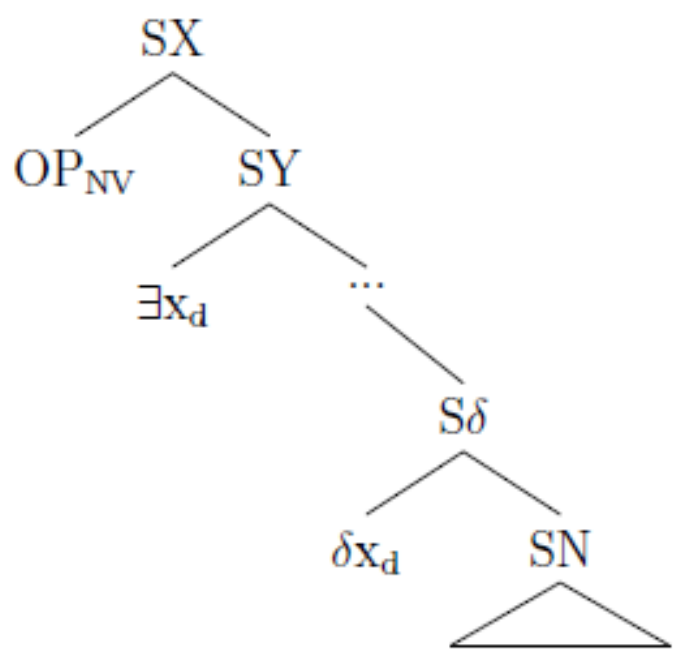

Esquemáticamente, resumimos en (54) las características del indefinido nulo $\delta$ :

(54) a. $\delta \mathrm{SN}$ es un indefinido de la forma $\mathrm{SN}\left(\mathrm{xd}_{\mathrm{d}}\right)$, en el que $\mathrm{xd}_{\mathrm{d}}$ es una variable de individuos dependiente.

b. Dado que $x_{d}$ es dependiente, no puede ser clausurado existencialmente a menos que haya un operador no verídico más alto que el existencial.

Cabe destacar que el hecho de que $\delta$ sea referencialmente deficiente no implica que nunca pueda introducir referentes discursivos. De hecho, sí puede hacerlo si el referente es insertado en un contexto subordinado y no en el principal. Consideremos nuevamente el ejemplo de (17a), repetido en (55). En este caso, libro mejor que "Cien años de soledad" no introduce un referente al contexto matriz, pero sí al subordinado por el predicado seminegativo dudar. De este modo, puede ser retomado por el pronombre $l o$, dado que este se encuentra bajo el alcance del mismo operador.

(55) Dudo que haya leído [ $\delta$ libro mejor que Cien años de soledad $]_{\text {i y }}$ no te loi haya recomendado.

Como se puede observar, la postulación del indefinido nulo $\delta$ nos permite conectar dos aspectos de estas construcciones que mencionamos previamente: la antiespecificidad y la legitimación en contextos no verídicos. Respecto a la primera cuestión, estos nombres desnudos nunca podrán tener una lectura específica debido a que $\delta$ es referencialmente deficiente. En cuanto al licenciamiento, el operador no verídico resulta obligatorio, ya que $\delta$ introduce una variable dependiente que solo puede ser clausurada existencialmente en estos entornos.

\section{CONCLUSIONES Y CUESTIONES PENDIENTES}

En este trabajo hemos analizado una construcción del español que ha recibido escaso interés en la bibliografía: los nombres contables en singular sin determinante bajo el alcance de la negación. A lo largo del artículo hemos abordado dos aspectos fundamentales: las condiciones de 
licenciamiento del nombre y su interpretación. Respecto a la primera cuestión, mostramos la inadecuación empírica de una explicación en términos de (pseudo)incorporación y propusimos que estos SSNN se comportan como términos de polaridad que se legitiman en contextos no verídicos. Este acercamiento nos permitió, además, diferenciar estos SSNN de las palabras-n y de las construcciones de alguno pospuesto. En relación a la segunda cuestión, sostuvimos que estos SSNN presentan una interpretación antiespecífica. Particularmente, se comportan como indefinidos referencialmente vagos. Por último, postulamos que estos SSNN se encuentran encabezados por un indefinido nulo $\delta$, que se caracteriza por ser referencialmente deficiente. De este modo, logramos vincular la necesidad de legitimación en contextos no verídicos y la interpretación antiespecífica.

Cabe señalar que la existencia de este tipo de indefinidos referencialmente deficientes ya ha sido reportada interlingüísticamente. Así, por ejemplo, se ha identificado en griego (kanenas; Giannakidou 1998), rumano (vreun; Farkas 2002), salish ( $k u$; Matthewson 1998), chino (shenme; Lin 1998) y coreano (nwuku-(ila)to; Giannakidou y Yoon 2016), entre otras lenguas. Si consideramos el paisaje general de los indefinidos, de acuerdo con la clasificación clásica de Haspelmath (1997), $\delta$ presentaría la distribución que muestra el gráfico de (56): como hemos visto, puede aparecer en interrogativas totales (35), en contextos de negación indirecta -predicados seminegativos como dudar (31), preposición $\sin (32)$, adverbios modales (33) y cuantificativos seminegativos (34)-, en casos de negación (30), y en el antecedente de un condicional (36).

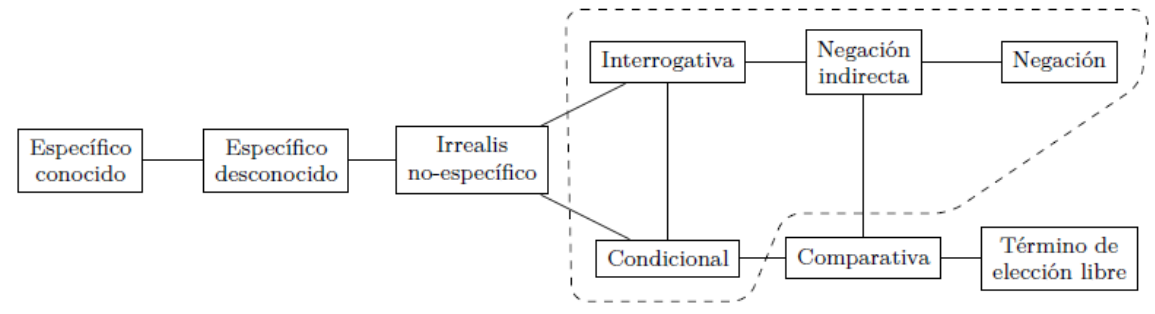

Vale aclarar, sin embargo, que el cuadro que presentamos en este trabajo está lejos de ser completo. Uno de los aspectos centrales de esta construcción que resta por explicar es el problema del modificador. Como señalamos en la sección 2, este tipo de estructuras requieren obligatoriamente la presencia de un modificador de carácter proposicional (e.g., una cláusula relativa).
a. *No leí libro.
b. No leí libro que me haya gustado.
c. *No leí libro atrapante.

En una primera aproximación, esta necesidad de que el nombre se encuentre modificado recuerda a los casos de subtrigging ${ }^{12}$ (LeGrand 1975, Dayal 1998). En inglés, el término any se licencia en contextos no verídicos, modales o genéricos. En este sentido, su aparición en una 
oración episódica como (58a) resulta agramatical. Sin embargo, su presencia se vuelve aceptable cuando el nombre se encuentra modificado por una cláusula relativa (58b). Cabe destacar que no cualquier modificador puede legitimar al término any: así, por ejemplo, la presencia del adjetivo prenominal angry en (58c) no influye en la gramaticalidad de la oración. Como es evidente, este patrón resulta muy similar, al menos superficialmente, al de los singulares desnudos en contextos no verídicos del español (57).
a. *Mary talked to any student.
b. Mary talked to any student who was angry.
c. *Mary talked to any angry student.

Si bien la obligatoriedad del modificador en los nombres escuetos aquí tratados parece asemejarse a los casos de subtrigging, consideramos que no se trata del mismo fenómeno. En primer lugar, como vimos previamente, en las estructuras de singulares desnudos, a diferencia de lo que ocurre en los casos de any, no basta con la presencia del modificador para licenciar al nombre, sino que es necesario además un entorno no verídico. En segundo lugar, los casos de subtrigging involucran términos de elección libre. Sin embargo, como mostramos anteriormente, los nombres desnudos que estudiamos se comportan como indefinidos referencialmente vagos.

Consideramos que una posible explicación al problema del modificador podría formularse en términos de selección. De este modo, el indefinido $\delta$ se combinaría únicamente con SSNN que ya resulten, en algún sentido, referencialmente deficientes. Esto último podría implementarse de diversas formas, por ejemplo, asumiendo que el nombre debe cumplir con una condición de fluctuación (Dayal 2009), mediante la postulación de individuos posibles (Dayal 1998), o a partir de las propiedades intensionales del subjuntivo (Giannakidou 2013). Cabe señalar que solo los modificadores proposicionales pueden satisfacer este tipo de condiciones. En cualquier caso, esta solución no deja de estar en el terreno de la especulación. Será motivo de futuros trabajos desentrañar este problema.

\section{Bibliografía}

Alonso Ovalle, L.; MenÉndez Benito, P. (2010): “Modal indefinites”, Natural Language Semantics 18(1), pp. 1-31. https://doi.org/10.1007/s11050-009-9048-4

BACH, E. (1982): "Purpose clauses and control", en P. Jacobson y G. Pullum (eds.): The nature of syntactic representation. Dordrecht: Reidel, pp. 35-57. https://doi.org/10.1007/978-94-009-7707-5 2

BAKER, M. (1988): Incorporation: A theory of grammatical function changing. Chicago: University of Chicago Press.

BAKER, M. C. (1996): The polysynthesis parameter. Oxford: Oxford University Press.

BAKER, M. C. (2014): "Pseudo noun incorporation as covert noun incorporation: Linearization and crosslinguistic variation", Language and Linguistics 15(1), pp. 5-46. https://doi.org/10.1177/1606822X13506154

BEninCÀ, P. (1980): “Nomi senza articolo”, Rivista di Grammatica Generativa Roma 5, pp. 51-63. 
BORIK, O.; GEHRKE, B. (2015): “An introduction to the syntax and semantics of pseudoincorporation”, en: O. Borik y B. Gehrke (eds.): The syntax and semantics of pseudo-incorporation. Leiden: Brill, pp. 1-43. https://doi.org/10.1163/9789004291089002

BORTHEN, K. (2003): Norwegian bare singulars. Tesis doctoral. Norwegian University of Science and Technology.

BoSQUE, I. (1980): Sobre la negación. Madrid: Cátedra.

BoSQUE, I. (1996): "Por qué determinados sustantivos son sustantivos indeterminados. Repaso y balance", en I. Bosque (ed.): El sustantivo sin determinación. La ausencia de determinante en la lengua española. Madrid: Visor, pp. 13-119.

BRUCART, J. M. (1994): "Syntactic variation and grammatical primitives in generative grammar", Lynx: Panorámica de estudios lingüísticos 4, pp. 145-176.

BRUCART, J. M. (1995): “Sobre más aditivo y los términos de polaridad negativa en español”. Manuscrito inédito.

BRUCART, J. M. (1999): “La estructura del sintagma nominal: las oraciones de relativo", en I. Bosque y V. Demonte (dirs.): Gramática descriptiva de la lengua española. Madrid: Espasa Calpe, vol. 1, pp. 395-522.

Costa, S. (2007): “Negación morfológica y polaridad negativa”, en: V. Bertolotti, S. Caviglia, S. Costa, M. Grassi, M. Malcuori y M. D. Muñoz Núñez (eds.): Estudios de lingüística hispánica. Cádiz: Servicio de Publicaciones de la Universidad de Cádiz, pp. 71-108.

DAYAL, V. (1998): "Any as inherently modal", Linguistics and Philosophy 21(5), pp. 433-476. https://doi.org/10.1007/s11049-011-9118-4

DAYAL, V. (2003): “A semantics for pseudo-incorporation”. Manuscrito. Rutgers University.

DAYAL, V. (2009). “Variation in English Free Choice Items”, en R. Mohanty y M. Menon (eds.): Universals and variation: Proceedings of GLOW in Asia VII. Hyderabad: EFL University Press, pp. 237-256.

DAYAL, V. (2011): “Hindi pseudo-incorporation”, Natural Language \& Linguistic Theory 29(1), pp. 123-167. https://doi.org/10.1007/s11049-011-9118-4

EsPiNAL, M. T.; MCNALLY, L. (2011): “Bare nominals and incorporating verbs in Spanish and Catalan”, Journal of Linguistics 47(1), pp. 87-128. https://doi.org/10.1017/S0022226710000228

EtXeberRia, U.; Giannakidou, A. (2017): “A unified analysis of Spanish algún/algunos: antispecificity, plurality, and NP-anaphora". Manuscrito inédito.

FARKAS, D. (2002). "Extreme non-specificity in Romanian”, en C. Beyssade, R. Bok-Bennema, F. Drijkoningen y P. Monachesi (eds.): Romance Languages and Linguistic Theory 2000. Amsterdam: John Benjamins, pp. 127-151. https://doi.org/10.1075/cilt.232.09far

FERNÁNDEZ RAMíREZ, S. (1951): Gramática española, vol. 3. Madrid: Revista de Occidente.

GeHrke, B.; LeKaKoU, M. (2013): “How to miss your preposition”, Studies in Greek Linguistics 33, pp. $92-106$.

Giannakidou, A. (1998): Polarity sensitivity as (non)veridical dependency. Amsterdam: John Benjamins. https://doi.org/10.1075/la.23

Giannakidou, A. (1999): "Affective dependencies", Linguistics and Philosophy 22(4), pp. 367-421. https://doi.org/10.1023/A:1005492130684

Giannakidou, A. (2001): “The meaning of free choice”, Linguistics and Philosophy 24(6), pp. 659-735. https://doi.org/10.1023/A:1012758115458 
GiAnNAKIDOU, A. (2006): "N-words and negative concord", en M. Everaert y H. van Riemsdijk (eds.): The Blackwell Companion to Syntax. Oxford: Blackwell, vol. 3, pp. 327-391. https://doi.org/10.1002/9780470996591.ch45

GianNAKIDOU, A. (2011): "Negative and positive polarity items”, en K. von Heusinger, C. Meienborn, y P. Portner (eds.): Semantics: and international handbook of natural language meaning. Berlín: Mouton de Gruyter, vol. 3, pp. 1660-1712.

GIANNAKIDOU, A. (2013): “(Non)veridicality, evaluation, and event actualization: Evidence from the subjunctive in relative clauses", en M. Toboada, y R. Trnavac (eds.): Nonveridicality and Evaluation. Theoretical, Computational, and Corpus Approaches. Leiden: Brill, pp. 17-49.

GIANNAKIDOU, A. (2018): "A critical assessment of exhaustivity for negative polarity items: The view from Greek, Korean, Mandarin, and English", Acta Linguistica Academica 65(4), pp. 503-545. https://doi.org/10.1556/2062.2018.65.4.1

GIANNAKIDOU, A.; QUER, J. (2013): "Exhaustive and non-exhaustive variation with free choice and referential vagueness: Evidence from Greek, Catalan, and Spanish", Lingua 126, pp.120-149. https://doi.org/10.1016/j.lingua.2012.12.005

GIANNAKIDOU, A.; Yoon, S. (2016): "Scalar marking without scalar meaning: Non-scalar, non-exhaustivity even-marked NPIs in Greek and Korean", Language 92(3), pp.522-556. https://doi.org/10.1353/lan.2016.0047

HALE, K.; KEYSER, S. J. (1998): “The basic elements of argument structure”, MIT Working Papers in Linguistics 32, pp. 73-118.

HASPELMATH, M. (1997): Indefinite pronouns. Oxford: Oxford University Press.

HEIM, I. (1982): The semantics of definite and indefinite pronouns. Tesis doctoral. University of Massachusetts, Amherst.

HeusingER, K. von (2011): “Specificity”, en C. Meinborn, K. von Heusinger y P. Portner (eds.): Semantics: An international handbook of natural language meaning. Berlin: Mounton de Gruyter, vol. 2, pp. 10241057.

KAMP, H. (1981): "A theory of truth and semantic representation", en J. Groenendijk, T. Janssen y M. Stokhof (eds.): Fromal Methods in the Study of Language. Amsterdam: Mathematical Centre, pp. 277-322.

KARTTUnen, L. (1976): “Discourse referents”, en J. McCawley (ed.): Syntax and Semantics 7: Notes from the Linguistic Underground. New York: Academic Press, pp. 363-385. https://doi.org/10.1163/9789004368859 021

LACA, B. (1999): "Presencia y ausencia de determinante", en I. Bosque y V. Demonte (dirs.): Gramática descriptiva de la lengua española. Madrid: Espasa Calpe, vol. 1, pp. 891-928.

LAKA, I. (1990): Negation in syntax: On the nature of functional categories and projections. Tesis doctoral. Massachusetts Institute of Technology.

LAPESA, R. (1996): “El sustantivo sin actualizador en español”, en I. Bosque (ed.): El sustantivo sin determinación. La ausencia de determinante en la lengua española. Madrid: Visor Libros, pp. 121-137.

LEGRAND, J. E. (1975): Or and any: The semantics and syntax of two logical operators. Tesis doctoral. University of Chicago.

LeonetTi, M. (1999): “El artículo”, en I. Bosque y V. Demonte (dirs.): Gramática descriptiva de la lengua española. Madrid: Espasa Calpe, vol. 1, pp. 787-890. 
LIN, J.-W. (1998): “On existential polarity-wh-phrases in Chinese”, Journal of East Asian Linguistics 7(3), pp. 219-255. https://doi.org/10.1023/A:1008284513325

MASSAM, D. (2001): "Pseudo noun incorporation in Niuean”, Natural Language \& Linguistic Theory 19(1), pp. 153-197. https://doi.org/10.1023/A:1006465130442

MASUlLO, P. J. (1992): Incorporation and Case Theory in Spanish: A crosslinguistic perspective. Tesis doctoral. University of Washington.

MASUlLO, P. J. (1996): “Los sintagmas nominales sin determinante: una propuesta incorporacionista”, en I. Bosque (ed.): El sustantivo sin determinación. La ausencia de determinante en la lengua española. Madrid: Visor Libros, pp. 169-200.

MATEU, J. (2002): Argument structure. Relational construal at the syntax-semantics interface. Tesis doctoral. Universitat Autònoma de Barcelona.

MatThEWSon, L. (1998): Determiner System and Quantificational Strategies. Evidence from Salish. Tesis doctoral. University of British Columbia

Mithun, M. (1984): “The evolution of noun incorporation", Language 60(4), pp.847-894. https://doi.org/10.1353/lan.1984.0038

MURASUGI, K. (2014): "Noun incorporation, nonconfigurationality, and polysynthesis", en A. Carnie, D. Siddiqi y Y. Sato (eds.): The Routledge Handbook of Syntax. London: Routledge, pp. 283-303.

PENKA, D. (2015): "Negation and polarity", en N. Riemer (ed.): The Routledge Handbook of Semantics. London: Routledge, pp. 303-319.

PÉREZ SALDANYA, M. (1999): “El modo en las subordinadas relativas y adverbiales”, en I. Bosque y V. Demonte (dirs.): Gramática descriptiva de la lengua española. Madrid: Espasa Calpe, vol. 2, pp. 3253-3322.

RAE y ASALE 2009 = REAL ACADEMIA ESPAÑOLA y ASOCIACIÓN DE ACADEMIAS DE LA LENGUA ESPAÑOLA (2009): Nueva gramática de la lengua española. Madrid: Espasa.

ROBERTS, C. (1989): "Modal subordination and pronominal anaphora in discourse", Linguistics and Philosophy 12(6), pp. 683-721. https://doi.org/10.1007/BF00632602

SÁNCHEZ LóPEZ, C. (1999): “La negación”, en I. Bosque y V. Demonte (dirs.): Gramática descriptiva de la lengua española, Madrid: Espasa Calpe, vol. 2, pp. 2561-2632.

WONDER, J. P. (1990): “Noun incorporation in Spanish”, Hispanic Linguistics 4(1), pp. 149-177.

\section{Notas}

* Quisiera agradecerles a Andrés Saab, a Ángela Di Tullio, a Carlos Muñoz Pérez, a Eugenia Sciutto, a Fernando Carranza, a Laura Kornfeld, a Laura Stigliano, a Romina Trebisacce y a dos revisores anónimos de Verba, que leyeron y comentaron versiones previas de este trabajo. También le agradezco al grupo de lingüística y filosofía del lenguaje de la Sociedad Argentina de Análisis Filosófico por sus valiosos aportes.

${ }^{1}$ Bosque (1996) señala otros contextos en los que es posible la presencia de un nombre discontinuo singular sin determinante: (i) con verbos intensionales (Estoy buscando/* pintando piso), (ii) en estructuras coordinadas (Compré lápiz y papel), (iii) en construcciones estereotipadas (Llevaba sombrero), (iv) con predicados existenciales relacionados con acontecimientos periódicos o previstos (Mañana hay examen), y (v) en titulares de prensa de algunos países iberoamericanos (Hombre muere tras ser brutalmente atropellado). Si bien en este trabajo no nos ocupamos de estos casos, creemos que ninguno de ellos puede ser explicado bajo el análisis aquí propuesto (i. e., como términos de polaridad licenciados en contextos no verídicos). Por el contrario, consideramos que algunas de estas configuraciones 
(particularmente, las de verbos intensionales, las de predicados existenciales y las construcciones estereotipadas) pueden ser analizadas bajo un enfoque pseudoincorporacionista (véase Espinal y McNally 2011).

${ }^{2}$ Cabe destacar que los SSNN analizados en este trabajo son altamente productivos y no se encuentran fosilizados. En este sentido, se deben distinguir de locuciones que presentan una estructura similar (i. e. Negación + Predicado + Nombre contable singular sin determinante + Modificador), tales como No dejar títere con cabeza o No dar puntada sin hilo, cuyo significado, al igual que los casos de (7), tampoco es composicional y su sintaxis es más rígida.

3 De acuerdo con Borthen (2003), los predicados de tener son aquellos que introducen una relación asimétrica coexistente entre dos argumentos, el poseedor y el poseído, en la cual el poseedor es superior al poseído, en términos de control, dependencia parte-todo, animacidad o punto de vista.

${ }^{4}$ Ligado a la cuestión de la referencialidad, Espinal y McNally observan que los nombres escuetos que ellas estudian no pueden controlar sujetos de cláusulas no finitas (i. e., PRO), e. g., *Busco pis per PRO ser compartit ('Busco piso para ser compartido'). De acuerdo con las autoras, esto se debe a que, por tratarse de nombres pseudoincorporados, son elementos discursivamente opacos y, en consecuencia, no poseen las propiedades referenciales necesarias para controlar a PRO. Sin embargo, consideramos que estos adjuntos de finalidad pueden resultar problemáticos a la hora de evaluar cuestiones relacionadas con el control. Como sostiene Bach (1982), en estos casos PRO no está controlado sintácticamente, sino que el sujeto implícito de la cláusula no finita se determina discursivamente. En este sentido, creemos que este tipo de ejemplos puede no resultar relevante a la hora de argumentar a favor de un análisis en términos de pseudoincorporación.

${ }^{5}$ Como observa correctamente uno de los revisores anónimos, estos ejemplos constituyen casos de subordinación modal (Roberts 1989), esto es, contextos en los cuales un SN indefinido se encuentra bajo el alcance de un operador intensional o de un cuantificador y, en consecuencia, es anafóricamente accesible solo para un elemento que también se encuentra bajo el alcance de ese operador o cuantificador, ya sea de forma explícita o implícita. Esto podría llevar a pensar que estos nombres sí resultan referencialmente opacos, dado que en muchos contextos no pueden ser retomados por un pronombre. Sin embargo, a nuestro entender, no lo son en el sentido en el que lo discuten Espinal y McNally. Como señalamos previamente, de acuerdo con las autoras, los nombres pseudoincorporados no pueden ser retomados por una anáfora discursiva porque no introducen argumentos, sino que funcionan como modificadores del verbo. Por el contrario, consideramos que en los ejemplos de (17) sí se introduce un antecedente discursivo. En este sentido, la opacidad referencial a la que se puede aludir en estos casos se debe a las restricciones de accesibilidad anafórica que imponen los respectivos operadores y no a las propiedades del SN. En la sección 5 daremos cuenta de este fenómeno a partir de la noción de variable dependiente (Giannakidou 2011).

${ }^{6}$ De aquí en más, todas las traducciones son nuestras.

${ }^{7}$ Como señala un revisor anónimo, en algunos dialectos esta oración resulta gramatical. En esos casos, la palabra-n nada funciona como un indefinido no negativo, esto es, algo.

${ }^{8}$ Cabe resaltar que este tipo de adverbios modales tienen un significado negativo. En este sentido, se diferencian de otros adverbios modales no negativos que, si bien dan lugar a entornos no verídicos, no legitiman este tipo de nombres desnudos (*Probablemente haya leído libro mejor que este). De este modo, consideramos que los contextos de legitimación de estos SSNN, además de ser no verídicos, deben contener un componente negativo, ya sea en términos semánticos o pragmáticos. Dejamos esta cuestión pendiente para futuras investigaciones.

9 Brucart $(1995,1994)$ propone que en estas construcciones la posición de Especificador del SN está ocupada por un operador nulo al que denomina TPN. Este operador permite interpretar al SN como un término de polaridad negativa, de manera tal que libro alguno se interpretaría como ningún libro. Dado su carácter negativo, el operador debe estar licenciado por la negación, lo cual explica el hecho de que en su ausencia la oración resulte agramatical (37b). De acuerdo con Brucart, la postulación de este operador permite explicar además por qué un SN no puede interpretarse como término de polaridad negativa si en su especificador se halla un elemento que no expresa explícitamente tal polaridad (e. g., No leyó algún libro).

${ }^{10}$ Como señalamos en la nota al pie 5 y en la sección siguiente, estos ejemplos constituyen casos de subordinación modal (Roberts 1989).

${ }^{11}$ Asumimos una semántica para los indefinidos en la línea de Heim (1982) y Kamp (1981).

${ }^{12}$ Agradezco a un revisor anónimo por haberme llamado la atención sobre este fenómeno. 